\title{
A FORCE MEASURING MICROSYSTEM BASES ON ELECTROTHERMAL V-SHAPED ACTUATOR
}

\author{
Pham Hong Phuc ${ }^{1, *}$ \\ ${ }^{1}$ Hanoi University of Science and Technology, Vietnam \\ *E-mail: phuc.phamhong@hust.edu.vn
}

Received 09 March 2020 / Published online: 22 August 2020

\begin{abstract}
This paper describes design and calculation of an electrothermal V-shaped actuator (EVA) and an amplification mechanism integrated into a force measuring microsystem (FMMS), aims to apply for characterization of a micro beam. Displacement and driving force are generated by thermal expansion of the V-shaped silicon beams while applying a voltage to the electrodes of the EVA. ANSYS simulation helps to find out the relations between thermal force and displacement corresponding to driving voltage and determine the temperature of $\mathrm{V}$-shaped beam at various applying voltages. In our simulation, with applying voltage $U_{m}=38$ volt for six pairs of $V$-shaped beam, the maximal temperature of the beam reaches approximately to $1100^{\circ} \mathrm{C}$ and causes a melting phenomenon of the silicon beam. The additional amplification mechanism allows actuator's displacement to be 6 times larger than before the improvement, thus the bending deformation of the micro beam can be seen perfectly, i.e. the force loading on the beam can be computed more exactly via a measured displacement of the beam tip. In addition, this FMMS has smaller size and supplies a larger beam's deformation at the same voltage in comparison with previous design.
\end{abstract}

Keywords: SOI-MEMS technology, V-shaped micro actuator, test beam, bending deformation.

\section{INTRODUCTION}

Almost micro-electro-mechanical system (MEMS) devices work under periodic loading and stress. As a result, the micro structures can be failed after a specific working time [1-3]. Recent years, determining exactly a force/moment loading on the micro structure such as a beam, spring or micro membrane has become a controversial topic as well as an interesting challenge in MEMS.

There are many researches on the lifetime and force-measurement of the micro actuator in various aspects. They cover main topics such as: new structure, integrated measuring system, or application of smart materials, etc. Naraghi and Chasiotis [4] proposed an integrating system to test the strength of nano wire using five electrothermal 
V-shaped actuators in order to grip and pull them. In [3], Huy et al. performed experiments, evaluated loading distribution and failure of silicon specimens based on Weibull and Paris law, proposed the method for predicting the critical force that causes failure of MEMS structure with different surface's roughness [5].

Determining force/load in micro system is an interesting problem for MEMS designers and researchers because it is difficult to measure perfectly value of micro/nano forces [6-8]. Moreover, their values may be changed while operating because of the periodic voltage or random load.

The authors in $[9,10]$ used static ruler to measure deformation of the elastic beam (both fixed-fixed and cantilever beams) and may calculate the electrostatic force acting on the beam due to relation between load and deformation of the beam. But a tiny deformation of the beam (i.e. a few of micrometer), which causes a significant measuring error, is a drawback of using this structure.

In this work, we propose a design of new force measuring microsystem (FMMS), which will be fabricated by using a SOI-MEMS technology with an aim to examine and evaluate the actuated-force acting on a cantilever test beam in micro devices. Here, the electrothermal V-shaped actuator (EVA) with six-pair-beams is used as a driving component, force is generated by thermal expansion of the thin beams will transmit and act on the middle of the test beam (3) (see Fig. 1). A lever (2) helps to amplify displacement of contact point (i.e. lever tip) is 6 times larger than displacement of a shuttle (1). Therefore, deformation of test beam can be measured more exactly via a ruler (5).

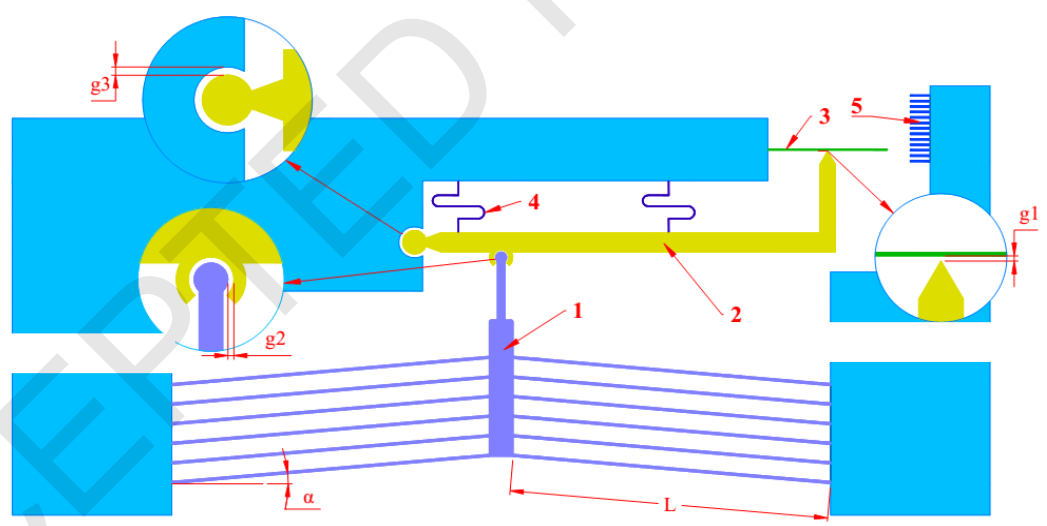

Fig. 1. Configuration of the force measuring microsystem (FMMS)

\section{CONFIGURATION AND WORKING OF THE SYSTEM}

\subsection{Configuration}

Fig. 1 shows the configuration of the FMMS, which consists of one V-shaped actuator as a moving part (a shuttle (1) at the center is suspended by 6 pairs of symmetric Vbeam), the end of shuttle connects to a lever (2) by an articulated joint, two springs (4), cantilever test beam (3) and the ruler (5). $g_{2}$ and $g_{3}$ are fabrication gaps between fixed and moving part of joint. $g_{1}=2 \mu \mathrm{m}$ is a safe gap from the tip of lever to the test beam. The 
symmetrical V-beams (with slope angle $\alpha=2^{\circ}$ have dimension as: Length $L=750 \mu \mathrm{m}$; width $b=6 \mu \mathrm{m}$; thickness $h=30 \mu \mathrm{m}$. Lever (2) has a function as a linear magnification mechanism with a horizontal length $L_{2}=1200 \mu \mathrm{m}$ (Fig. $5)$, the distances from the shuttle tip to the articulated joint on the left and tip of lever on the right are $200 \mu \mathrm{m}$ and $1000 \mu \mathrm{m}$ respectively, gives an displacement amplifying ratio of 6 . The ruler (5) consists of twenty parallel lines with the pitch of $5 \mu \mathrm{m}$.

The elastic springs (4) which connected to the lever (2) will push it back to an initial position while V-shaped actuator is not working. Fig. 2 describes the geometric dimension of

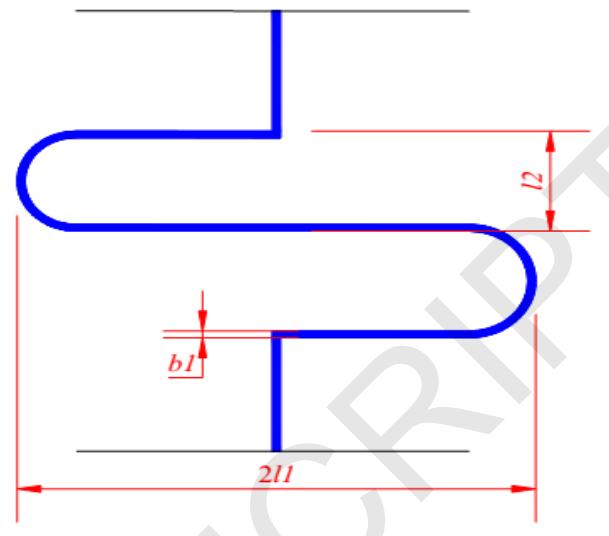

Fig. 2. Dimension of the spring spring, where $2 l_{1}=150 \mu \mathrm{m}$ is the width of the spring, $l_{2}=20 \mu \mathrm{m}$ is the pitch and $b_{1}=$ $3 \mu \mathrm{m}$ is the thickness of spring's wire.

\subsection{Working principle}

When applying a $D C$ voltage on two fixed electrodes of the actuator, an electric current transmits through the thin beam and generates a thermal emission. The V-shaped beam system will be heated and expanded, then drives the shuttle (1) moving up in a vertical direction. After overcoming the gap $g_{2}$, the shuttle pushes the lever (2) turning around its joint center and amplifies displacement of lever tip up to 6 times in comparison with displacement of shuttle. As a result, the test beam (3) is bended at the middle by the force actuated from the lever tip. The larger displacement of tip of the test beam can be measured easily due to the ruler (5) attached in substrate. Value of force acts on the test beam can be calculated by using a load-displacement formula of the cantilever beam, which will be mentioned at the next section.

If the voltage goes to zero, the temperature of beams will decrease gradually. The beams will shrink and pull the shuttle returns to the initial position. The lever will turn back because of spring force.

\section{CALCULATION OF FORCE AND DISPLACEMENT}

\subsection{Relation between the force and deformation of cantilever test beam}

While the force $P$ acts on the middle point $B$ of the test beam (Fig. 3), a bending stress at the connected point A can be expressed by

$$
\sigma_{\max }=\frac{M_{A}}{W}=\frac{3 P L_{1}}{h b^{2}}
$$

or

$$
P=\frac{\sigma_{\max } h b^{2}}{3 L_{1}}
$$


where $L_{1}=300 \mu \mathrm{m}, b=3 \mu \mathrm{m}$ and $h=30 \mu \mathrm{m}$ are the length, width and thickness of the test beam correspondingly. Via Eq. (2), with replacing flexural strength of silicon $\sigma_{m}=$ $3000 \mathrm{MPa}$ [11], we find out a maximum (or critical) force $P_{\max }$ that can load on the test beam is about $0.9 \mathrm{mN}$.

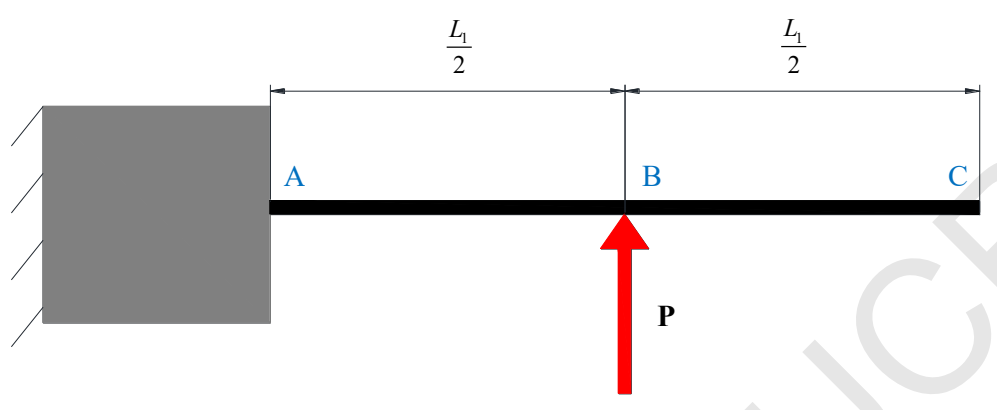

Fig. 3. Force acting on the test beam

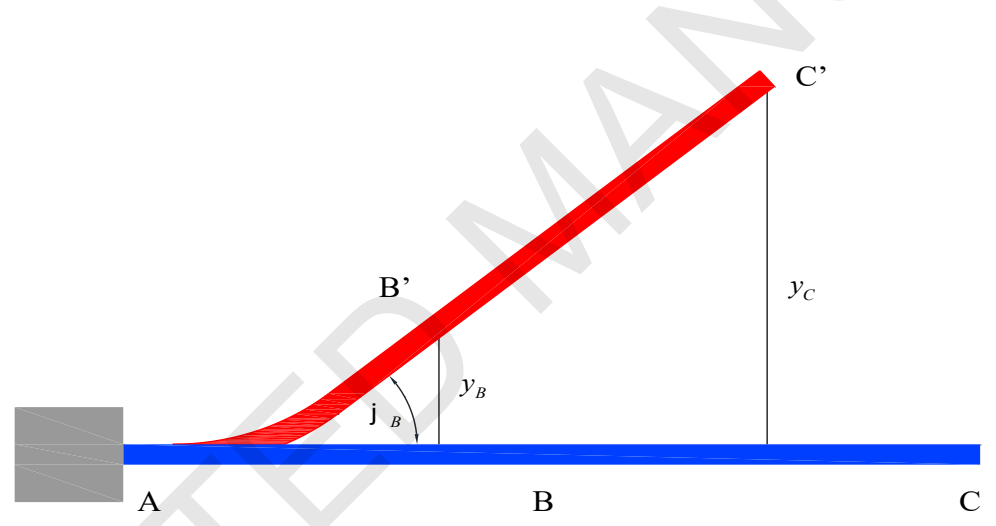

Fig. 4. Deformation of point B and tip C of the test beam

Here, a friction force between the lever tip and test beam while contacting is perpendicular to force $P$ and do not cause the bending of the test beam. Thus, it can be ignored.

According to the strength of materials theory, a skew deformation $y_{B}$ and angular deformation $\varphi_{B}$ of the middle point B in Fig. 4 can be computed by

$$
\begin{aligned}
y_{B} & =\frac{P L_{1}{ }^{3}}{24 E I^{\prime}} \\
\varphi_{B} & =\frac{P L_{1}^{2}}{8 E I},
\end{aligned}
$$

where $E=169 \mathrm{GPa}$ is a Young's modulus of silicon; $I$ is an inertial moment of beam cross-section. Assuming that line $\mathrm{B}^{\prime} \mathrm{C}^{\prime}$ is linearity, we can express a deformation $y_{C}$ as

$$
y_{C}=y_{B}+\frac{L_{1}}{2} \sin \left(\varphi_{B}\right)=y_{B}+\frac{L_{1}}{2} \sin \left(\frac{3 y_{B}}{L_{1}}\right)=\frac{P L_{1}^{3}}{24 E I}+\frac{L_{1}}{2} \sin \left(\frac{P L_{1}^{2}}{8 E I}\right) \text {. }
$$


From Eqs. (3)-(5), if know the value of $y_{C}$ (via checking the ruler (5) by microscope), we can calculate easily a deformation $y_{B}$ and the force $P$. As an example: assuming that measured deformation $y_{C}=60 \mu \mathrm{m}$, we get the deformation $y_{B}=24.14 \mu \mathrm{m}$ and the force $P$ is about $0.245 \mathrm{mN}$.

\subsection{Relation between driving force of V-shaped actuator and deformation of test beam}

Fig. 5 shows the schematic view of forces acting on the lever while working. Here, and are elastic forces of the left and right springs; $P^{\prime}=P$ is reaction force from the test beam; $F_{1}$ is a driving force of the actuator loading on the lever. We have the equilibrium equation of moment around the articulated joint as followed

$$
\frac{1}{6} L_{2} F_{1}=\frac{1}{10} L_{2} F_{l x 1}+\frac{3}{4} L_{2} F_{l x 2}+L_{2} P
$$

and the driving force of the actuator is

$$
F_{1}=\frac{3}{5} k y_{1}+\frac{9}{2} k y_{2}+6 P .
$$

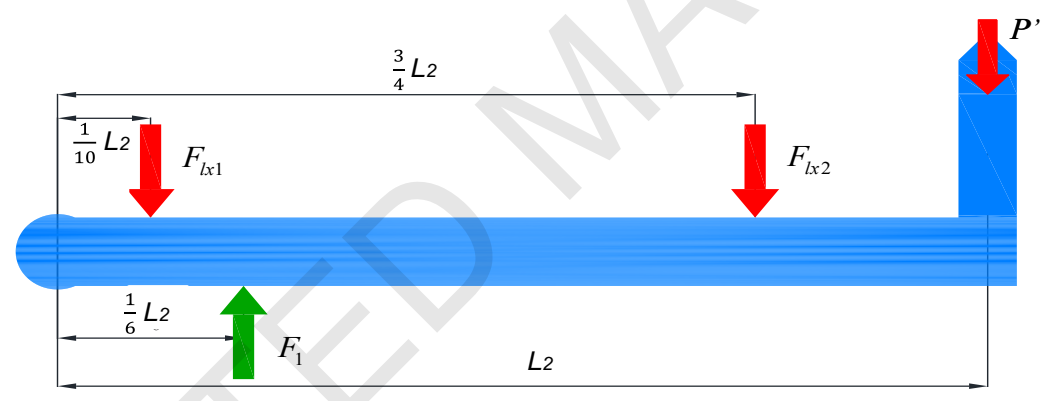

Fig. 5. Forces act on the lever

By substituting (3) into (7), we have

$$
F_{1}=\frac{3}{5} k\left[\frac{1}{10}\left(y_{B}+g_{1}\right)\right]+\frac{9}{2} k\left[\frac{3}{4}\left(y_{B}+g_{1}\right)\right]+6 y_{B} \frac{24 E I}{L_{1}^{3}},
$$

where $k=15.23 \mu \mathrm{N} / \mu \mathrm{m}$ is a stiffness of each spring obtained by simulation; and are the deformation of left and right springs. Eq. (8) describes a relation between driving force $F_{1}$ and deformation $y_{B}$ at the middle of test beam. If $y_{B}=24.14 \mu \mathrm{m}, g_{1}=2 \mu \mathrm{m}$ and $P$ $=0.245 \mathrm{mN}$ as mentioned above, we need the driving force of $\mathrm{V}$-shaped actuator: $F_{1} \approx$ $2.838 \mathrm{mN}$. Required displacement $y_{F 1}$ of the shuttle can be calculated as

$$
y_{F 1}=\left(\frac{y_{B}+g_{1}}{6}\right)+g_{2}
$$

where $g_{1}$ and $g_{2}$ are the fabrication gaps and described as in Section 2.1. If we choose $g_{1}=g_{2}=2 \mu \mathrm{m}$, the displacement of the shuttle should be equal or larger than $6.36 \mu \mathrm{m}$. 


\subsection{Simulation}

FEM simulation of V-shaped actuator model has been performed by using ANSYS in order to examine a relation between displacement of actuator $y_{F 1}$ and the driving voltage $U$ (Fig. 6(a)). Moreover, a temperature distribution along the V-beam at various driving voltages has been also considered to find out a limitation of temperature (i.e. maximal driving voltage $U_{m} \approx 38 \mathrm{~V}$ ) so as to avoid a melting phenomenon which can occur in a thin beam of the V-shaped actuator at a high voltage (Fig. 6(b)).

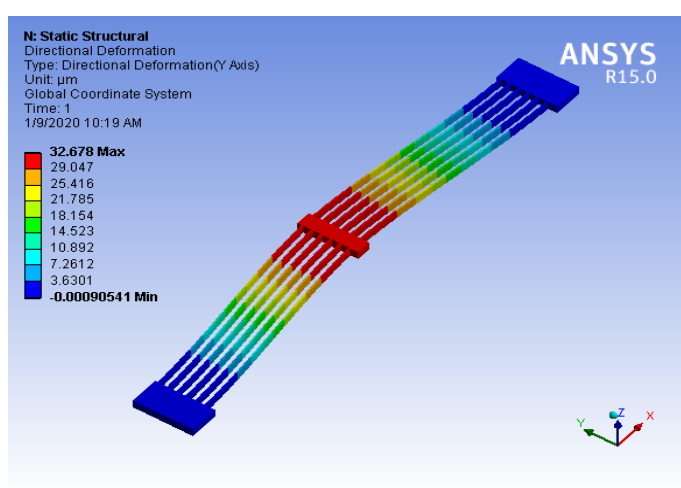

(a) Displacement depends on driving voltage

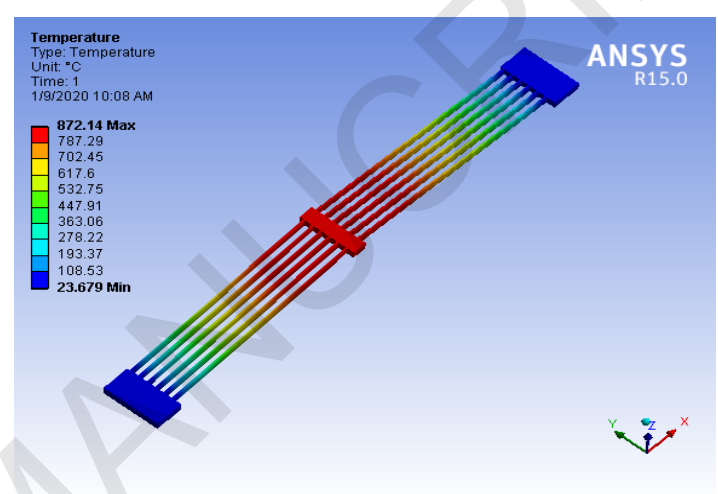

(b) Temperature distribution along the beam

Fig. 6. Simulation of the V-shaped actuator

Material properties of the silicon are listed in Tab. 1. Simulation results are summarized in Tab. 2. To continue above example, aimed to get the displacement of actuator $y_{F 1}=6.36 \mu \mathrm{m}$ (i.e. the deformation $y_{C}=60 \mu \mathrm{m}$ ), we need to apply a voltage of $10.9 \mathrm{~V}$ for V-shaped actuator (approximating 3.5 times smaller than the critical voltage $U_{m}$ as calculated above).

Table 1. Material properties of silicon $[9,11]$

\begin{tabular}{lcc}
\hline Young's modulus of silicon & $E(\mathrm{MPa})$ & $169 \mathrm{E}+3$ \\
\hline Mass density of silicon & $\rho\left(\mathrm{kg} / \mathrm{m}^{3}\right)$ & 2330 \\
Poisson's ratio & $v$ & 0.28 \\
\hline
\end{tabular}

Table 2. Values of actuator's displacement depends on driving voltage

\begin{tabular}{cccccccc}
\hline Driving voltage $U($ Volt $)$ & 10 & 10.9 & 12 & 14 & 16 & 18 & 20 \\
\hline Displacement $y_{F 1}(\mu \mathrm{m})$ & 5.39 & 6.36 & 7.43 & 9.72 & 12.17 & 14.82 & 17.58 \\
\hline
\end{tabular}




\section{CONCLUSIONS}

This paper presents design and calculation of new force measuring microsystem (FMMS) with the additional amplifying mechanism which allows to increasing the displacement of the test beam up to 6 times than before [10]. Results can be summarized as below:

Amplifying displacement of the lever tip can be measured more exactly by using the ruler located near the free end of the cantilever test beam. Furthermore, two springs connected to the lever can help it return to the initial position easier while the driving voltage goes to zero.

The dependence of the driving force of actuator $F_{1}$ as well as its displacement $y_{F 1}$ on the deformation of test beam $y_{B}$ is established in the equations from (6) to (9). In other words, knowing the value of $y_{C}$ enable to find $y_{B}$ and compute accurately the needed driving force $F_{1}$ loaded on the lever. Thus, a corresponding voltage applying for $\mathrm{V}$-shaped actuator will be determined.

Distribution of temperature along the thin beam has been solved by simulation. A critical driving voltage $U_{m}$ (in this case $U_{m} \approx 38 \mathrm{~V}$ ) is also proposed in order to avoid the failure of V-shaped beam.

Because of simple fabrication technology and low cost, this system with cover size of $2 \times 2\left(\mathrm{~mm}^{2}\right)$ can be developed and integrated to micro force-measurement device, not only for V-shaped actuator, but also for others such as electrostatic or piezoelectric actuator. Besides, it can be used widely to evaluate and check some mechanical properties of silicon or of other materials (example: failure load and allowable bending/tensile/compressive stress) in micro devices.

\section{ACKNOWLEDGMENT}

This research is funded by Vietnam National Foundation for Science and Technology Development (NAFOSTED) under grant number 107.01-2019.05.

\section{REFERENCES}

[1] P. H. Pham, D. V. Dao, S. Amaya, R. Kitada, and S. Sugiyama. Straight movement of micro containers based on ratchet mechanisms and electrostatic comb-drive actuators. Journal of Micromechanics and Microengineering, 16, (12), (2006), pp. 2532-2538. https://doi.org/10.1088/0960-1317/16/12/003.

[2] D. V. Dao, P. H. Pham, and S. Sugiyama. Multimodule micro transportation system based on electrostatic comb-drive actuator and ratchet mechanism. Journal of Microelectromechanical Systems, 20, (1), (2010), pp. 140-149. https://doi.org/10.1109/jmems.2010.2090503.

[3] V. L. Huy, S. Kamiya, J. Gaspar, and O. Paul. Statistical characterization of fracture strength and fatigue lifetime of polysilicon thin films with different stress concentration fields. Journal of Solid Mechanics and Materials Engineering, 6, (11), (2012), pp. 1013-1029. https://doi.org/10.1299/jmmp.6.1013.

[4] M. Naraghi and I. Chasiotis. A mems platform for mechanical testing of soft nanofibers and nanowires. Technical report, University of Illinois at Urbana Champaign, (2007).

[5] V. L. Huy, S. Kamiya, K. Nagayoshi, H. Izumi, J. Gaspar, and O. Paul. A prediction scheme of the static fracture strength of MEMS structures based on the characterization of damage 
distribution on a processed surface. Journal of Micromechanics and Microengineering, 23, (4), (2013). https://doi.org/10.1088/0960-1317/23/4/045008.

[6] H. Conrad, H. Schenk, B. Kaiser, S. Langa, M. Gaudet, K. Schimmanz, M. Stolz, and M. Lenz. A small-gap electrostatic micro-actuator for large deflections. Nature Communications, 6, (1), (2015), pp. 1-7. https://doi.org/10.1038/ncomms10078.

[7] A. M. Ousaid, S. Haliyo, S. Régnier, and V. Hayward. Micro-force sensor by active control of a comb-drive. In 2013 IEEE/ASME International Conference on Advanced Intelligent Mechatronics, IEEE, (2013), pp. 612-617. https://doi.org/10.1109/aim.2013.6584160.

[8] P. H. Phuc, D. K. Toan, D. B. Lam, N. T. Khoa, and N. T. Dung. Design and fabrication of the trapezoidal electrostatic comb-drive actuator. Vietnam Journal of Mechanics, 34, (4), (2012), pp. 261-269. https://doi.org/10.15625/0866-7136/34/4/2338.

[9] P. H. Pham, T. K. Dinh, L. B. Dang, K. T. Nguyen, and D. V. Dao. Micro cam system driven by electrostatic comb-drive actuators based on SOI-MEMS technology. Microsystem Technologies, 21, (3), (2015), pp. 699-706. https://doi.org/10.1007/s00542-014-2086-y.

[10] P. H. Pham, K. T. Nguyen, and L. B. Dang. Design and performance of a high loading electrostatic micro linear motor. Microsystem Technologies, 21, (11), (2015), pp. 2469-2474. https://doi.org/10.1007/s00542-014-2392-4.

[11] J. J. Allen. MEMS design. Taylor \& Francis Group, LLC, (2005). 